\title{
Design of a training tool for improving the use of hand-held detectors in humanitarian demining
}

\author{
Roemi Fernández
}

Héctor Montes

Carlota Salinas, Pablo González de Santos and Manuel Armada

\begin{abstract}
Purpose - The purpose of this paper is to introduce the design of a training tool intended to improve deminers' technique during close-in detection tasks. Design/methodology/approach - Following an introduction that highlights the impact of mines and improvised explosive devices (IEDs), and the importance of training for enhancing the safety and the efficiency of the deminers, this paper considers the utilization of a sensory tracking system to study the skill of the hand-held detector expert operators. With the compiled information, some critical performance variables can be extracted, assessed, and quantified, so that they can be used afterwards as reference values for the training task. In a second stage, the sensory tracking system is used for analysing the trainee skills. The experimentation phase aims to test the effectiveness of the elements that compose the sensory system to track the hand-held detector during the training sessions.

Findings - The proposed training tool will be able to evaluate the deminers' efficiency during the scanning tasks and will provide important information for improving their competences.

Originality/value - This paper highlights the need of introducing emerging technologies for enhancing the current training techniques for deminers and proposes a sensory tracking system that can be successfully utilised for evaluating trainees' performance with hand-held detectors.
\end{abstract}

Keywords Training, Tracking, Explosives, Detection, Tracking systems, Scanning, Humanitarian demining, Hand-held detectors

\section{Introduction}

Landmines, cluster munitions, explosive remnants of war (ERW) and improvised explosive devices (IED) are an enduring legacy of conflict. These devices can remain active for decades, they are not aware of negotiation or peace treaties and do not distinguish between soldiers and civilians. In 2010 , a total of 4,191 new landmine casualties were reported, 5 percent more than in 2009 , and a total of 72 states, as well as seven disputed areas, were confirmed or suspected to be mine-affected (Landmine and Cluster Munition Monitor, 2011). The problem of hidden IEDs has become especially worrying. The number of such bombings has increased from close to zero a decade ago to more than 4,000 per year in Afghanistan alone (Miles et al., 2012).

Apart from the human casualties, the presence of landmines also produces negative economic effects, as it denies access to the affected areas and their resources, causing deprivation and social problems among the affected populations. Therefore, the elimination of antipersonnel mines is a vital requirement for the recovery of the affected regions (Habib, 2002). In military demining the objective is to clear a minefield as fast as possible using brute force, and usually a clearance rate of $80-90$ percent is accepted (Rosengard et al., 2001). On the other hand, humanitarian demining is more difficult and dangerous, as it requires the complete removal of all mines, ERWs and IEDs and

The authors acknowledge funding from the European Community's Seventh Framework Programme (FP7/2007-2013 TIRAMISU) under Grant Agreement No. 284747 and partial funding under Robocity 2030 S-0505/DPI-0176 and FORTUNA A1/039883/11 (Agencia Española de Cooperación Internacional para el Desarrollo - AECID). Dr Roemi Fernández acknowledges support from CSIC under grant JAE-DOC. Dr Héctor Montes acknowledges support from Universidad Tecnológica de Panamá and from CSIC under grant JAE-DOC. 
the return of the cleared minefield to normal use (Hussein and Waller, 2000; Shimoi, 2002; Gonzalez de Santos et al., 2007; Baudoin et al., 2011).

Metal detectors are utilised as the principal equipment for detection since the Second World War due to its simplicity and affordability. This generic technology is usually composed of a search head, containing one or more coils carrying a timevarying electric current. This current generates a corresponding time-varying magnetic field which propagates towards the metallic target in order to induce eddy currents in it, which in turn generate a detectable magnetic field (Guelle et al., 2003). The substantial metal content of earlier generation mines made their detection quite easy. However, landmine design has evolved in response. Contemporary landmines are built with plastic bodies and miniscule amounts of metal or no metal at all, vastly increasing the difficulty of detection for metal detectors (Habib, 2011).

In order to alleviate this problem, a number of new hand-held mine detectors are emerging as alternatives to the traditional ones (Habib, 2011). An example is the Hand-held Standoff Mine Detection System (Doheny et al., 2005), the US Army's AN/PSS-14 dual sensor, hand-held mine detector capable of detecting metallic and non-metallic anti-tank and antipersonnel mines by combining an electromagnetic induction sensor, ground penetrating radar (GPR) and sophisticated algorithms. The Minehound VMR3 from Vallon is also a dual sensor that combines the same aforementioned technologies for the detection of metal and metal-free mines and IEDs (Daniels and Curtis, 2006; Daniels et al., 2007). However, GPR still presents some limitations. Natural subsurface inhomogeneities (such as roots, rocks, and water pockets) can cause the GPR to register return signals that resemble those of landmines and thus are a source of false alarms. In addition, GPR performance can be highly sensitive to complex interactions among mine metal content, interrogation frequency, soil moisture profiles, and the smoothness of the ground surface boundary. Such complex interplays make performance highly variable and difficult to predict (MacDonald and Lockwood, 2003).

A variety of possible chemical methods for detecting low concentrations of explosives in air or in soil samples have also been investigated recently. Some years ago, Nomadics Inc. developed landmine hand-held detectors based on the principle of trace/vapour detection (GICHD, 2006). Perhaps the greatest obstacle that these systems must overcome is the need for a probability of detection of 1.0 if they are to replace manual prodders for confirming the presence of mines. Another problem is that the presence of explosive residues in soil from sources other than landmines will trigger false alarms, which restricts their use in battlefield environments. These detectors are also limited by incomplete knowledge of how explosive vapours migrate in the shallow subsurface (MacDonald and Lockwood, 2003).

Nuclear quadrupole resonance (NQR) technology has also been combined with hand-held metal detectors in order to provide a next step in mine detection. An NQR device induces an radio frequency (RF) pulse of an appropriate frequency in the subsurface via a coil suspended above ground. This RF pulse causes the explosives' nuclei to resonate and induce an electrical potential in a receiver coil. The major weakness of NQR is the fact that, because of its nuclear properties, trinitrotoluene (TNT), which comprises the explosive fill of most landmines, provides a very weak signal, posing a formidable signal to noise ratio problem. Another significant limitation is the susceptibility of NQR to RF interference from the environment. This is especially problematic for TNT detection because the frequencies required to induce a response from TNT $(790-900 \mathrm{kHz})$ are in the AM radio band (MacDonald and Lockwood, 2003; GICHD, 2006).

The design of innovative accurate sensors may contribute to reduce the amount of time needed to determine whether a landmine exists, but does not increase the safety of the deminers. The proficiency of landmine detection equipment operators is highly variable. Expert operators of the most commonly used hand-held detectors exhibit mine detection probabilities of 0.9 (Staszewski, 1999), but a simple mistake can jeopardize their lives. In 2010, the number of casualties among humanitarian clearance operators was double than the ones recorded for 2009. There were 131 deminer casualties ( 36 deminers killed; 95 injured) recorded in 15 states/areas in 2010 , compared to 67 deminer casualties in 2009 (Landmine and Cluster Munition Monitor, 2011).

Training is then one of the most crucial aspects in order to improve the safety and effectiveness of the mine detection activities performed by the human operators. Training is not only required for novice operators but also when new detection technologies are introduced. For instance, the proper use of dual sensors requires more training and practice compared to metal detectors (Takahashi and Gülle, 2010). Retraining is also essential for maintaining a high level of efficiency. Deminers who do not conduct humanitarian demining operations uninterruptedly during the period of two years are obliged to attend additional training courses (Laura, 2011). Some studies also indicate that the poor retention of operator skills is a common problem that endangers the success of the countermine operations and jeopardizes the personnel involved. A decline in the proportion of mine simulants detected was observed with as little as 30 days without practice. Solving this problem involves improving training to prevent or minimize such decrements from occurring, and developing intervention to restore performance to requisite levels as efficiently and as economically as possible through refresher training (Hancock, 2006).

However, in the literature there are very few studies that have been dedicated to the improvement of training techniques. The research undertaken by the Carnegie Mellon University in collaboration with the Army Research Laboratory, Human Research and Engineering Directorate Field Element stands out. In this research, an operator with extensive experience and an impressive record was recorded on video while his skills were tested in mine lanes with the AN/PSS-12 detector. After analysing 30 hours of video, his technique was translated into procedures that could provide an efficient training program for soldiers. Engineer soldiers that were given 15 hours of instruction based on these procedures, improved their detection rate from 15 to 87 percent (Davison et al., 2001; Staszewski, 2006). This significant improvement in the detection rates by using techniques and procedures qualitatively different from those traditionally taught confirms the need of incorporating an instruction based on the scientific knowledge rather than on personal introspection and intuitions of the training designers.

The Carnegie Mellon University has also developed a Sweep Monitoring System (SMS) for training tasks (Herman and Iglesias, 1999; Herman et al., 2000). The system employs two video cameras to track the movement of a target through space. The SMS records the position of the target at all times during 
the sweep and later replays the recorded data via a graphical interface, showing the resulting sweep coverage area to the observers. Nevertheless, the target used in the SMS is a big, brightly coloured foam ball mounted atop the detector (Daniel et al., 2004). Hence the detector does not closely resemble a real landmine hand-held detector.

This paper presents a training tool for analysing with the final goal of improving the use of hand-held detectors in humanitarian demining. The tool consists of a human machine interface, and a hand-held detector sensory tracking system (Fernández et al., 2011). The training tool can be easily adapted to be used with different kinds of handheld detectors. The purpose of the proposed tool is twofold:

1 The study of the expert's skills by quantifying some critical performance variables, so that they can be used later as reference values for the training tasks.

2 The efficiency evaluation of novice operators during the training tasks with hand-held detectors in order to give them feedback about important information for improving their competencies.

Therefore, the proposed tool will enable the development and implementation of instructions based on the scientific analysis of the problem and the formative and summative assessment of trainees. The emphasis in this article will be put on the testing of the elements that composes the hand-held detector sensory tracking system. The rest of the paper is organized as follows. Section 2 describes the sensory tracking system of the training tool. Section 3 explains the projected methodology for representing the knowledge acquired by the sensory tracking system. Section 4 presents the preliminary experimental results and finally, Section 5 summarises major conclusions.

\section{Hand-held detector sensory tracking system}

The sensory system approach of the training tool is based on an omnidirectional catadioptric stereo system, a time of flight (TOF) camera and a hand-held detector which is mounted with two inertial measurement units (IMUs), and several LEDs of high intensity utilised as landmarks for motion tracking. The proposed vision system provides a $360^{\circ}$ field of view (FOV) and stereo capability without sacrificing the image resolution too much. This is a great advantage in comparison with conventional imaging systems that are severely limited in their FOV. Moreover, acquisition of redundant information provides knowledge of the environment with greater clarity, precision and reliability. The TOF camera provides $3 \mathrm{D}$ imaging at a high frame rate, simultaneously providing intensity data and range information for every pixel. The IMUs have an accurate full $360^{\circ} 3 \mathrm{D}$ orientation output and a highly dynamic response combined with long-term stability. An overview of the main elements that compose the sensory tracking system is shown in Figure 1. A brief description of these elements is presented in the following subsections.

\subsection{External sensors}

The omnidirectional catadioptric stereo system and the TOF camera form the set of external sensors for the training tool. A catadioptric system is the result of combining a refracting (dioptric) surface with a reflecting (catoptrics) one in a carefully arranged configuration to capture a much wider FOV. Thus, a catadioptric system is normally a compound of a conventional high-resolution camera acting as the refracting part and a curved mirror as the reflecting one. In order to acquire a single image
Figure 1 Overview of the multisensory tracking system Multisensory Tracking System

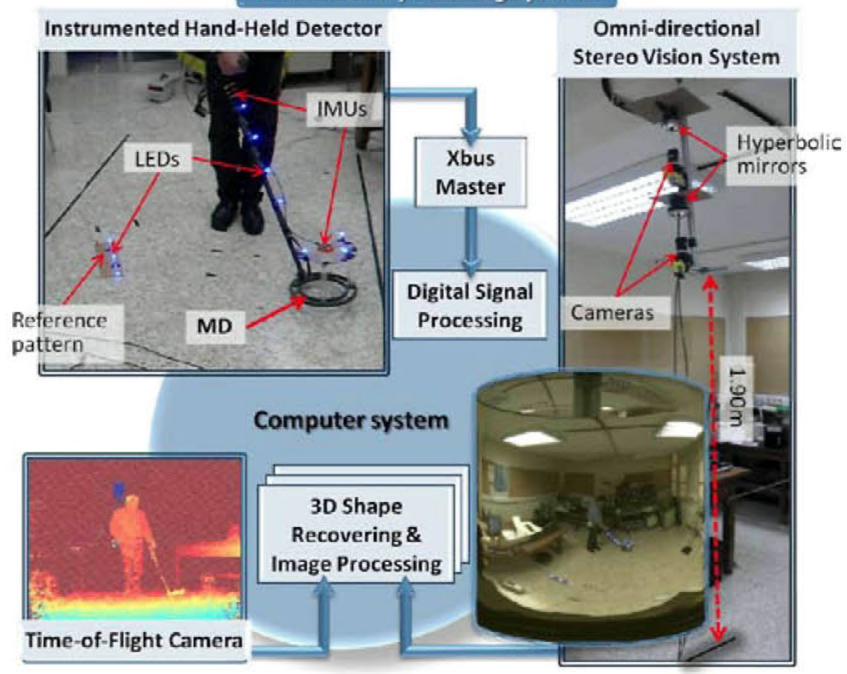

containing the information of the whole scene, the camera and the mirror must be arranged in a configuration so that the entire system has a single effective viewpoint (Baker and Nayar, 1999). Therefore, a single viewpoint is a requirement for the generation of pure perspective images from the sensed images. To generate omnidirectional images, perfect quadratic surfaces are considered as the only candidates for mirror shapes. In this way, every incidental ray of light that strikes the surface towards the mirror focus is reflected on the second focus. Since the geometry of the system is known, it is possible to compute the ray direction for each pixel and its irradiance value.

An omnidirectional catadioptric stereo system is usually constructed in one of the following ways (Benosman and Kang, 2001; Zhihui et al., 2008):

- Two decoupled high-resolution omnidirectional catadioptric systems are installed horizontally, and the axes of the imaging systems are vertical with the horizontal plane. However, there are occlusions between these two systems, which limit the FOV of the stereo vision system.

- Two decoupled high-resolution omnidirectional catadioptric systems are installed vertically on the same vertical axes. In this type of systems, the mirror can be parabolic or hyperbolic. However, when the system uses paraboloids as reflecting mirrors, it requires using expensive telecentric lens.

- Finally, an omnidirectional stereo vision system can be built by using two reflecting mirrors and a high-resolution camera. This type of stereo imaging system requires the two images of stereo vision to be captured with a single camera, which limits the imaging resolution.

For the proposed training tool, the stereo rectified configuration accomplished with two decoupled high-resolution omnidirectional catadioptric systems vertically aligned would be the most suitable ones. Using this type of design, there would not be any occlusions between the two systems, and it ensures that there is a parallax on $360^{\circ} \mathrm{FOV}$. Thus, the rectification process provides epipolar radial lines onto the image plane and when they are projected onto the panoramic perspective they become parallel lines to the vertical axis. As a result, the depth is isotropic in all directions. Figure 2 shows a close up of the omnivision 
Figure 2 Omnivision system

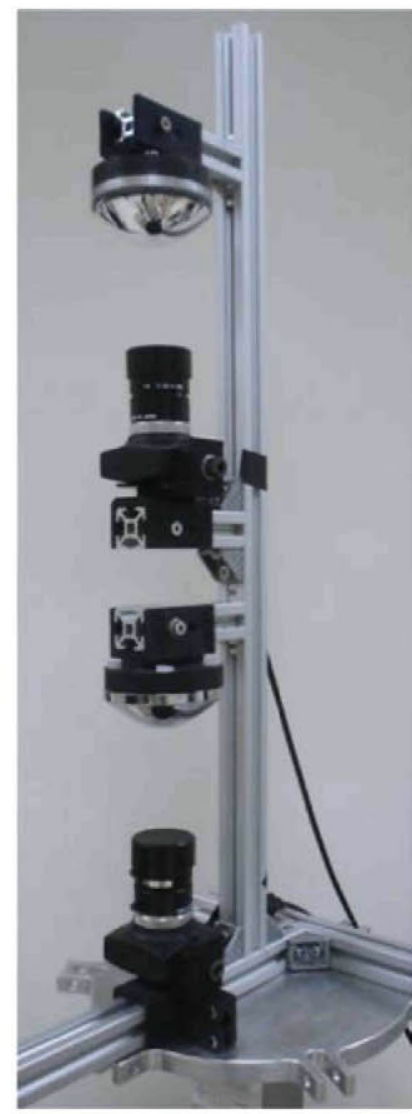

(a)
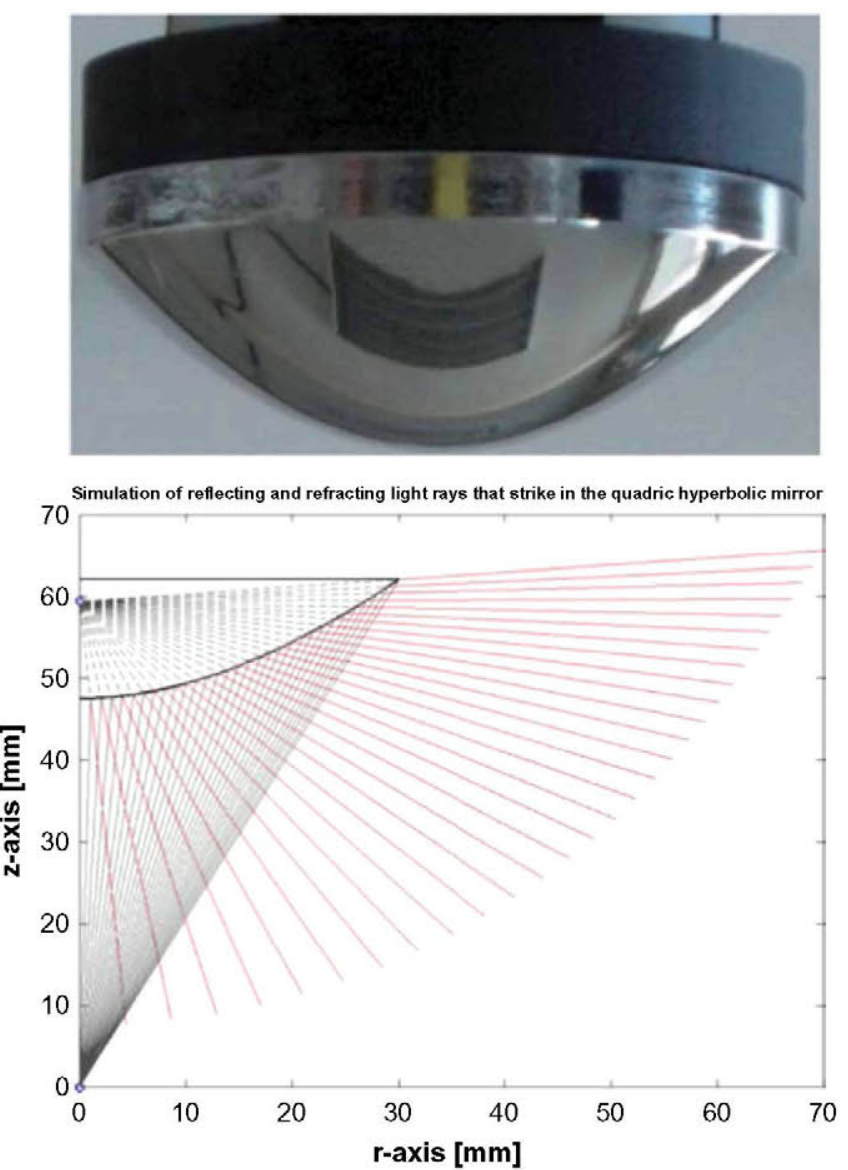

(b)

Notes: (a) Omnidirectional catadioptric stereo configuration; (b) hyperbolic mirror specially manufactured by the $\mathrm{CNC}$ micro-mechanisation and simulation of the reflecting ray lights that strike the mirror surface

stereo system, the hyperbolic mirror that has been designed and manufactured using the CNC micro-mechanisation facilities at the Centre for Automation and Robotics CSIC-UPM, and a simulation of the mirror profile (Salinas et al., 2011).

The TOF camera emits a near infrared modulated light $(850 \mathrm{~nm})$ that is reflected by the objects in the scene and sensed by a pixel array carried out in a specialised mixed CCD and CMOS process: the target depth is estimated by measuring the phase shift of the signal round-trip from the device to the target and back (Oggier et al., 2004). Therefore, the TOF camera provides a depth map, a grey level image, the $3 \mathrm{D}$ coordinates of the points cloud representing the acquire scene and a confidence map (the reliability of each measurement based on amplitude). Compared to stereo vision, the depth map is available without heavy calculations, since no correspondences between images pairs have to be established.

\subsection{Hand-held detector instrumentation}

The metal detector that has been selected as hand-held detector for the experiments in this study is the AN-19/2 manufactured by Schiebel (Guelle et al., 2003). However, the training tool can be easily adapted to be utilised with any kind of hand-held detector. The chosen device is able to detect mines with a very small metal content below the surface of the ground and in fresh or salt water. Its search head consists of concentric transmitting and receiving coils embedded in a plastic head. The length of the telescopic pole is adjustable and can be locked in three fixed positions. For the experiments, the length is fixed at $1,145 \mathrm{~mm}$. A light plastic plate is connected parallel to the search head with the objective of using it as a support for the sensors. The separation of the plastic plate from the sensor head was calculated for eliminating any chance of interference on the metal detector.

Several LEDs of high intensity are placed along the handheld structure: three on the light plastic plate that is above the search head and four on the wand. As they are attached by means of plastic clamps, they can be easily installed and removed. Figure 3 shows the distribution of the LEDs. With these landmarks, the omnivision stereo system is able to detect and track the position and orientation of both the wand and the search head during the sweeping motions.

Two MTx motion trackers (IMUs) from the company XSens Technologies B.V. (www.xsens.com) complete the proposed sensory system. The MTx is a small and light weight measurement unit that with a highly dynamic response provides drift-free and accurate 3DOF orientation (pitch, roll, 
Figure 3 Scheme and general view of the AN-19/2 metal detector with high-intensity LEDs and motion trackers
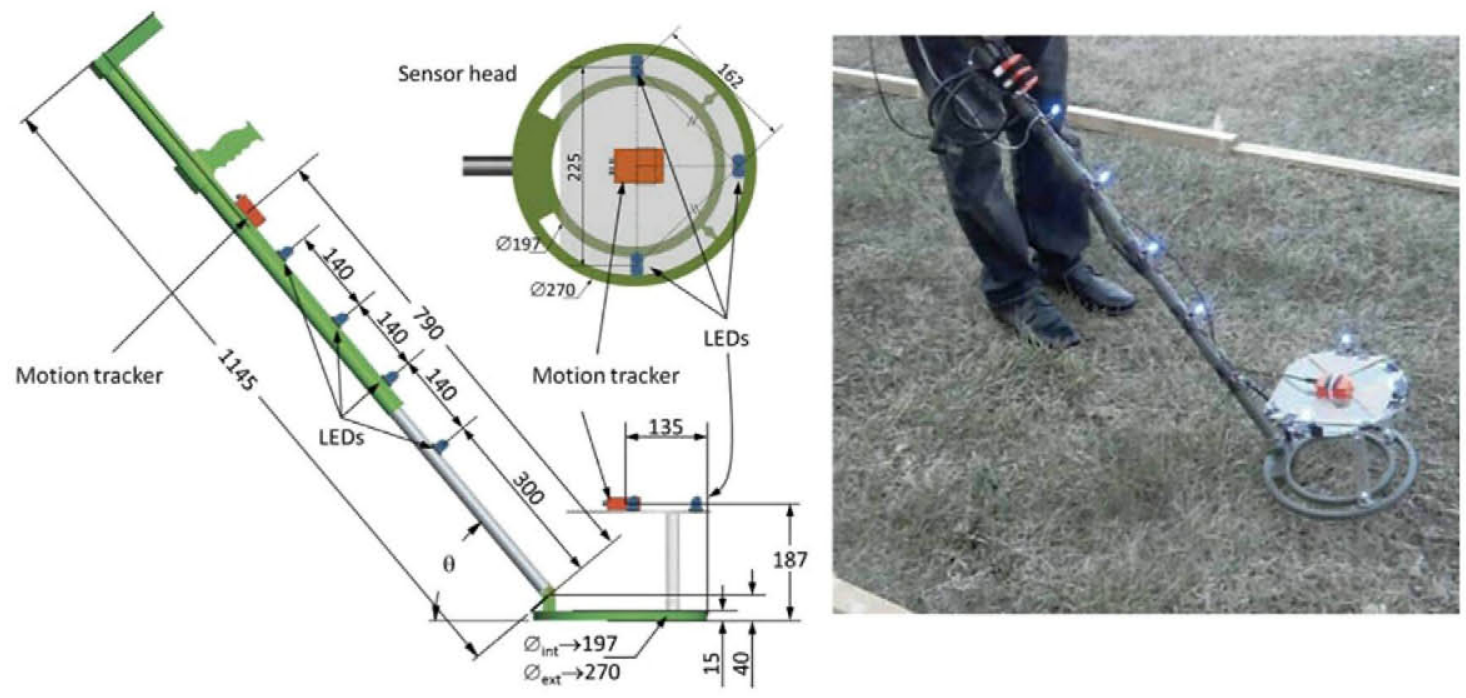

and yaw), as well as kinematic data: 3D acceleration, 3D rate of turn (rate gyro) and 3D earth-magnetic field. It is configured to output data from each triad of accelerometers, gyroscopes and magnetometers at $100 \mathrm{~Hz}$. One of these units is installed at the centre of the light plastic plate that is above the search head while the second is located in the wand at $790 \mathrm{~mm}$ from the joint that links the search head with the wand.

\section{Methodology for representing the acquired knowledge}

The sensory tracking system described above will be utilized for acquiring information in two different scenarios (Figure 4): when the expert's skills are studied in order to quantify some critical performance variables and when the efficiency of the deminers is evaluated during the training tasks.
Previous analysis of the expert's hand-held detection activities shows that their techniques and strategies differ from conventionally taught operating procedures. Whereas other operators perform detection on the basis of auditory outputs pointing out the existence of conductive materials, experts use the onset and offset of outputs that occurred during sweeping motions of the hand-held detector to create spatial patterns that they compare to learned models (Staszewski, 2004). They also modify their detection techniques to adapt to environmental variations (deserts, hilly rocky terrain, lands with a wide variety of vegetation). For this reason, the projected methodology proposes the utilization of the sensory tracking system to study the experts' skills in different environments and with different kinds of hand-held detectors. With the compiled information, some critical performance variables will be extracted, assessed, and quantified off-line, so that they can be used later

Figure 4 Overview of the proposed methodology

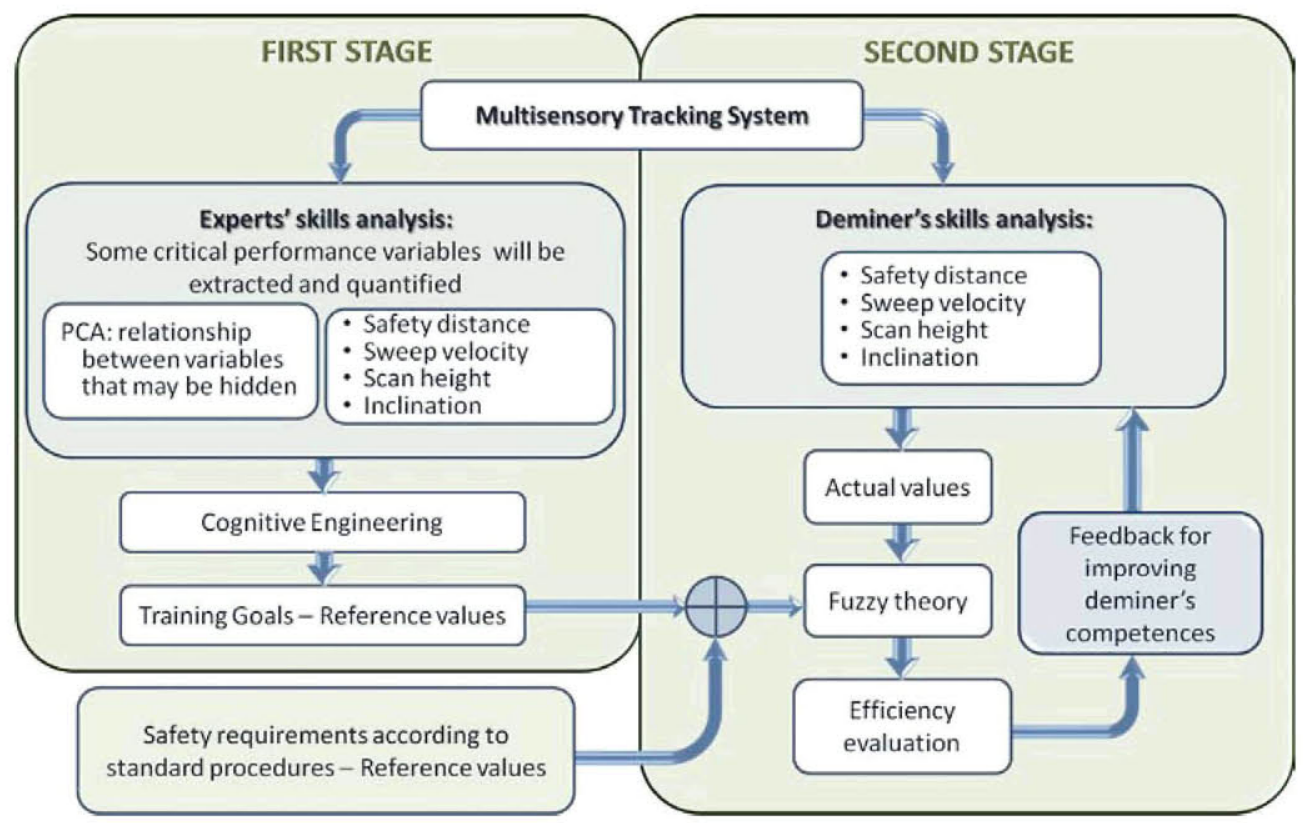


as reference values for the training task. The critical variables chosen preliminarily that will be analysed are the safety distance to advance the detector search-head on each sweep, the sweep velocity, the scan height, and the inclination of the hand-held detector head with respect to the ground. Principal component analysis (PCA) could also be used to explore the behavioural data. PCA is a well-established, standard approach for reducing the dimensionality of a large dataset, in order to identify any structure in the relationships between the variables that might otherwise be hidden. Therefore, PCA could highlight those underlying components that explain the most variance in the datasets as a whole. Cognitive engineering has proved to be a proper approach for solving applied problems in which human performance depends upon the quality of participants' thinking and skills. Cognitive models of expert skills have been successfully adopted for training in quite different domains such as medicine (surgery and dentistry) (Hegarty et al., 2007), aviation (Sarter and Amalberti, 2009) and landmine detection (Davison et al., 2001; Staszewski, 2006). Therefore, cognitive engineering will be used for translating the information acquired from the expert performance into modelling targets for unified, integrative theories of intelligence. In this way, instruction will be based on scientific knowledge rather than on personal introspection and intuitions of the training designers.

For the analysis and assessment of the trainee skills, the same set of critical variables proposed to parameterise the experts' performance is acquired and compared in real time with the reference training goals. In this way, the training tool is able to evaluate the deminers' efficiency during the scanning tasks online and give them feedback about important information for improving their competences. However, this evaluation can also be carried out off-line, recording the whole performance of the deminer, analysing it and finally, producing a report indicating the success of the learning process and identifying the points that should be enhanced. If the experts' skills have been studied previously, the reference training goals required for the assessment will be obtained from the results of the experts' performance. In case experts are not available for these experiences, trainee operators will be evaluated according to the reference values established in the standard procedures.

Standard procedures define some minimal requirements for the sweeping technique that should be followed in order to ensure the safety of the deminers. For the AN-19/2 metal detector these requirements are:

- Each sweep across the lane must overlap the previous sweep by about one-half the width of the detector head to ensure full coverage of the area being searched. Otherwise, a gap is left between sweep paths and mines can be missed. This is especially true for low-metal mines, which emit a very small electric field, often less than the width of the detector head.

- Operators' manual recommends a sweep rate of $1 \mathrm{~m} / \mathrm{s}$. However, if low-metal mines are going to be detected, this sweep velocity could be too fast. In this case, the detector head should be swept no faster than about $30 \mathrm{~cm} / \mathrm{s}$.

- Height head above the ground is the most important factor. The search of the metal detector should be moved not more that $5 \mathrm{~cm}$ about the ground. The closer the detector head is to the ground, the deeper the electrical field is projected and the greater is the possibility to detect the mines.

- When sweeping, the search head of the detector must remain horizontal to the ground at all times. If the detection task is being carried out on an irregular surface, the search head should be kept parallel to and at a constant height from the ground, following the variations in the surface.

- To prevent interferences, the distance between different search heads should not be less than $2 \mathrm{~m}$.

Since the fuzzy set theory has proved to be a worthwhile means for handling and representing experience-based heuristic knowledge, it will be used to represent the training goals. Given a universe of discourse $\mathbf{U}$, a fuzzy value $\mathbf{A}$ is defined by means of a possibility distribution $\pi^{\mathbf{A}}$ defined over $\mathbf{U}$ (Zadeh, 1975). Given a precise value $\mathbf{x} \in \mathbf{U}, \pi^{\mathbf{A}}(x) \in[\mathbf{0}, \mathbf{1}]$ represents the possibility of $\mathbf{A}$ being precisely $\mathbf{x}$. The possibility distribution $\pi^{\mathbf{A}}$ could be represented by means of a trapezoidal function specified by four parameters. In this way, $\mathbf{A}=(\alpha, \beta, \gamma, \delta), \boldsymbol{\alpha} \leq \boldsymbol{\beta} \leq \boldsymbol{\gamma} \leq \boldsymbol{\delta}$, where $[\beta, \gamma]$ represents the core, $\operatorname{core}(\mathbf{A})=\left\{\mathbf{x} \mid \pi^{\mathbf{A}}(\mathrm{x})=1\right\}$, and $] \alpha, \delta[$ represents the support, $\operatorname{support}(\mathbf{A})=\left\{\mathbf{x} \mid \pi^{\mathbf{A}}(\mathbf{x})>0\right\}$. The minimum and maximum values acceptable for the chosen critical variables are the beginning and the ending of the distribution support, respectively. In this way, for instance, if the deminer advances the hand-held detector with a lower velocity than the value corresponding with the beginning of the support, the scanning task is too time-consuming. If the deminer advances the handheld detector with a higher velocity than the one corresponding with the ending of the support, the sweep is being carried out too fast for efficient and safe landmine detection. The beginning and ending of the core are the interval limits where the ideal values lie. If $\mathbf{v}_{\mathbf{i}}$ is the sweep velocity output in $\mathrm{m} / \mathrm{s}$, the degree to which the training objectives are being achieved is $\pi^{\mathbf{A}}\left(\mathbf{v}_{\mathbf{i}}\right)$. If $\pi^{\mathbf{A}}\left(\mathbf{v}_{\mathbf{i}}\right)=0$ then the acquired velocity is outside the range of ideal values. Conversely, if $\pi^{\mathbf{A}}\left(\mathbf{v}_{\mathbf{i}}\right)=1$, the acquired velocity is within the range of ideal values. The closer $\pi^{\mathbf{A}}\left(\mathbf{v}_{\mathbf{i}}\right)$ is to 1 , the closer the sweep velocity output is to the ideal values.

Operators will be trained first indoors, utilising all the components of the sensory tracking system and in a training set composed entirely of simulant mines that replicate characteristics found in many landmines, but that do not represent any specific mine. In this way novice operators may familiarise themselves with the equipment and their response to the target mines. Once this first step is completed, the second stage involves training in field conditions in order to teach operators the adaptation to different environments (rainy, humid, hot) and types of soils (laterite, pure sand, sand mixed with magnetite, humus soil). In this phase, only motion trackers will be employed and tests will be blind, that is, operators will not know the locations and types of objects. Inert or drill mines instead of simulant mines will be adopted in accordance with the requirements of IMAS $10.50 \mathrm{~S} \& \mathrm{OH}$ for storage, transportation and handling of explosives. After a satisfactory level of competence is achieved, it may permissible to continue further training in hazardous areas, with real ammunition. Such training should only be conducted under close supervision and only with the approval of the National Mine Action Authorities. Special attention should also be given to IMAS 10.70 S\&OH for protection of the environment. Performance parameters will be analysed continuously during all the sessions. The evolution of these parameters will give important information for the study of other factors such as distraction, fatigue or stress during training. For instance, if some performance parameters leave the normal limits after a long period of good behaviour it could mean a symptom of distraction or fatigue. Stress conditions could be detected by comparing the performance of the operator against inert mines 
and real ammunition. After training, the last step will be the evaluation of the achievements. The metrics for measuring the efficiency of the training include the detection probability (fraction of encountered mines that are detected), the false alarm rate (the number of false alarms per square meter of test lane) and the probability of false positives (fraction of characterised clutter that is detected).

\section{Experimental results}

The main goal of the experimental phase was to evaluate the effectiveness of the sensory system to track the hand-held detector during the training sessions. For that reason, several tests were carried out in both laboratory and infield conditions.

The first stage involved the validation of the omnidirectional catadioptric stereo vision system. Figure 5 shows a representative pair of images selected from a large image sequence acquired with the omnidirectional catadioptric stereo image system and the detection results of the high-intensity LEDs that are present on the scene. Note that not only the LEDs that are placed in the hand-held detector structure are detected but also the three LEDs that form the reference pattern utilised for recovering $3 \mathrm{D}$ information from the environment. Several steps are required to accomplish this detection. First, an object segmentation based on the shape and the colour of the LEDs is carried out. Then the detected LEDs that form the hand-held detector wand are clustered if they fit a lineal model.
Finally, the detected LEDs that belong to the search head are clustered if they fit a triangle shape model.

After verifying that it is possible to use the stereo vision system to detect the position and orientation of the hand-held detector in space, a search sweep session is started. To simulate a real demining field scenario, a training lane is prepared. This lane is free from metal contamination and is $1.5 \mathrm{~m}$ width and has a length of $3 \mathrm{~m}$ in laboratory and $7 \mathrm{~m}$ outdoors. Figure 6 shows several pairs of images acquired during the sweep session while Figure 7 shows the tracking results of the hand-held detector structure in the image plane with the omnivision system during the scanning motions.

These results confirm that with the omnivision system it is feasible to detect and track a dynamic object without the necessity of moving the cameras. Moreover, the omnidirectional system enables the training tool to be aware of its complete surroundings at all times. The omnivision system has no problem in simultaneously detecting several hand-held detectors in distinctly different parts of its environment. In this way several operators can be evaluated simultaneously.

The second stage of testing concerns the use of the TOF camera. Several depth maps obtained with the TOF camera during the search sweep session are shown in Figure 8. From these range data and with proper algorithms a 3D reconstruction of both the operator and the hand-held detector can be accomplished. TOF camera makes data extraction simpler and quicker than the omnivision system, reducing

Figure 5 Image pair acquired with the omnidirectional catadioptric stereo system and the detection results of the high-intensity LEDs that are present in the scene
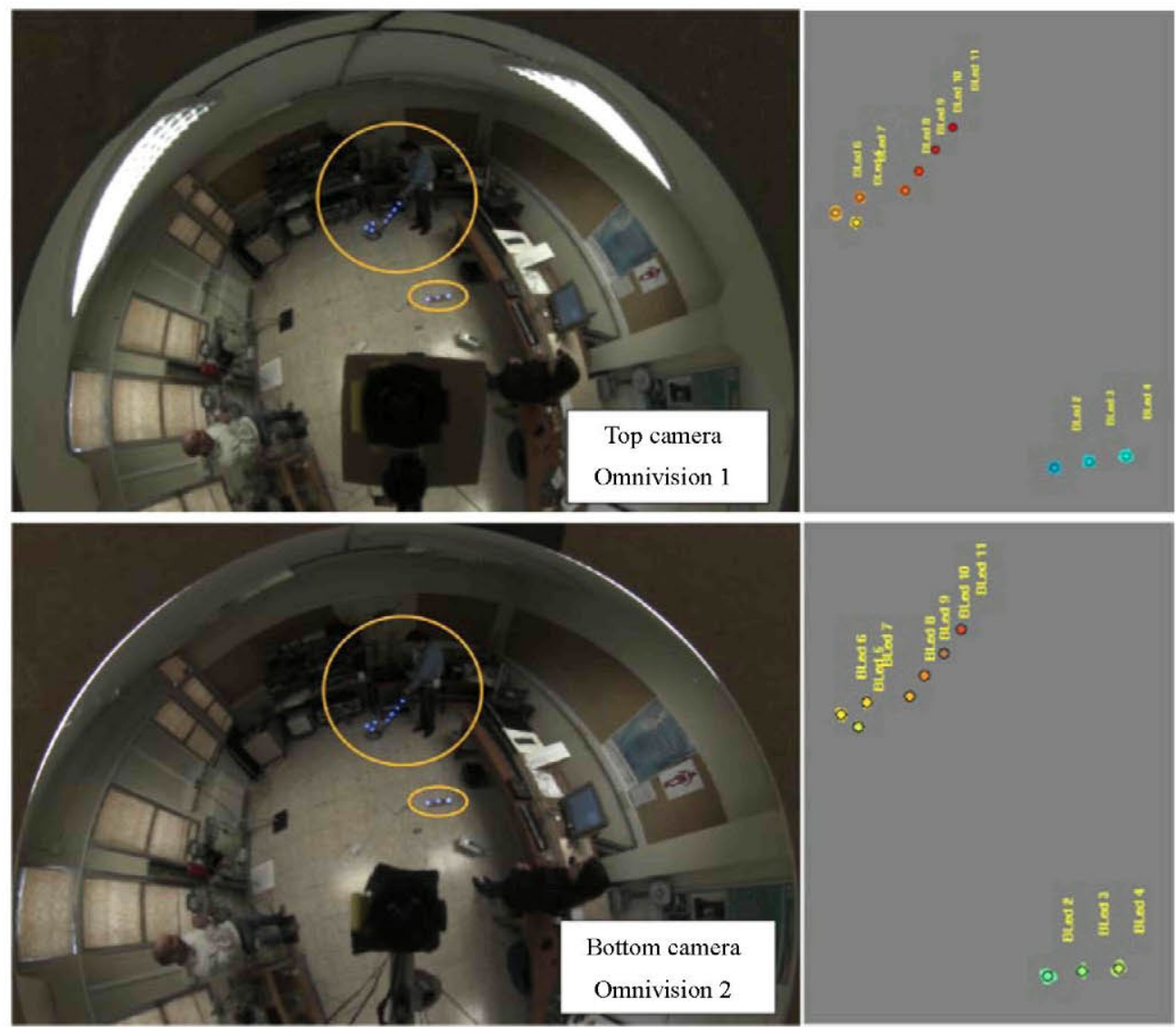
Figure 6 Pairs of images acquired with the omnidirectional catadioptric stereo system during the sweep session
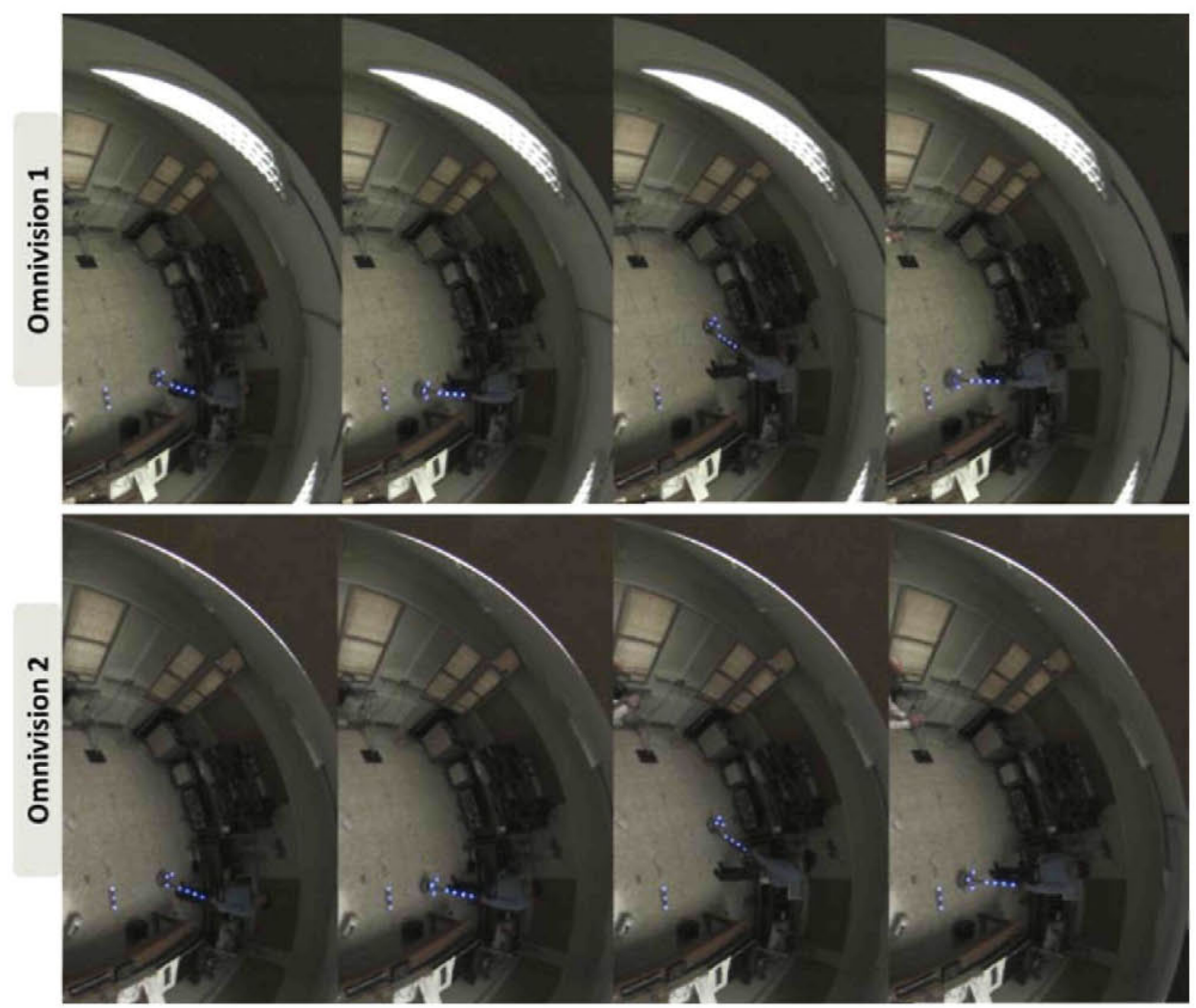

Figure 7 Tracking of the hand-held detector with the omnidirectional catadioptric stereo system

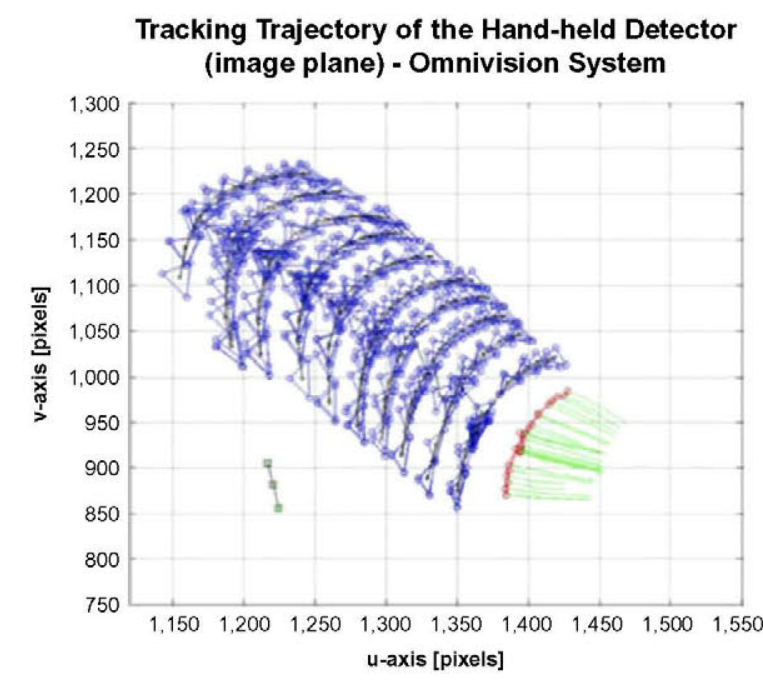

power consumption and computational time. Thus, a TOF camera proves to be especially adequate for real-time operation.

The third stage considers the use of the IMUs. Figure 9 shows the yaw, pitch and roll angles provided by the motion trackers in real time during the sweep session. Solid lines represent the signals corresponding to the search head and dashed lines to the wand of the metal detector. Yaw angles allow us to easily follow the sequence of sweep motions carried out in the ground plane. For instance, in the results obtained in this experiment, the sequence starts in the middle of the trajectory with a movement of the search head towards the left, followed by a complete
Tracking Trajectory of the Hand-held Detector (image plane) - Omnivision System 2

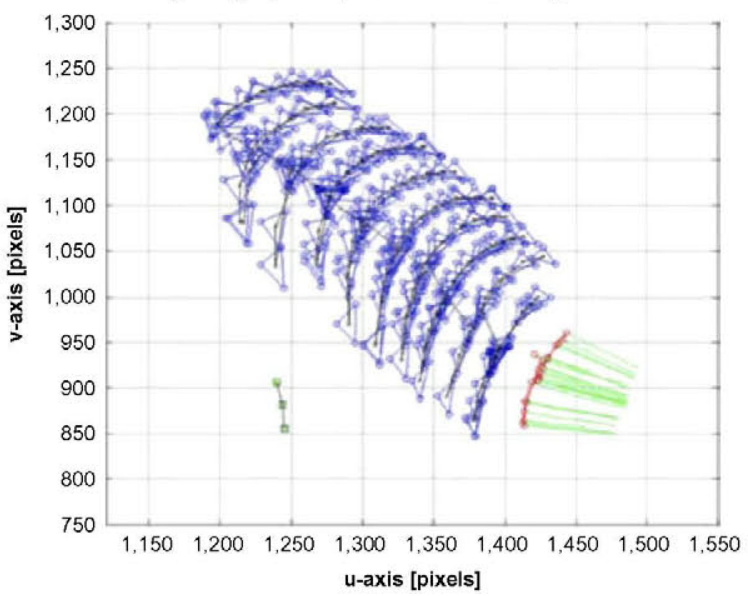

sweep of the lane to the right, a sweep to the left until the middle of the trajectory and a step to the front for the advancement. Pitch and roll angles can provide information about the elevation and inclination of the search head with respect to an initial reference position. In the results obtained in the presented experiment, pitch angles remain practically constant for both the search head and the metal detector wand during the whole session, except for some oscillations that occur approximately in the middle of the sequence. These oscillations are due to a series of irregularities present in the ground, where the operator is forced to reorientate the sensor head to keep it 
Figure 8 Depth maps obtained with the TOF camera during the search sweep session
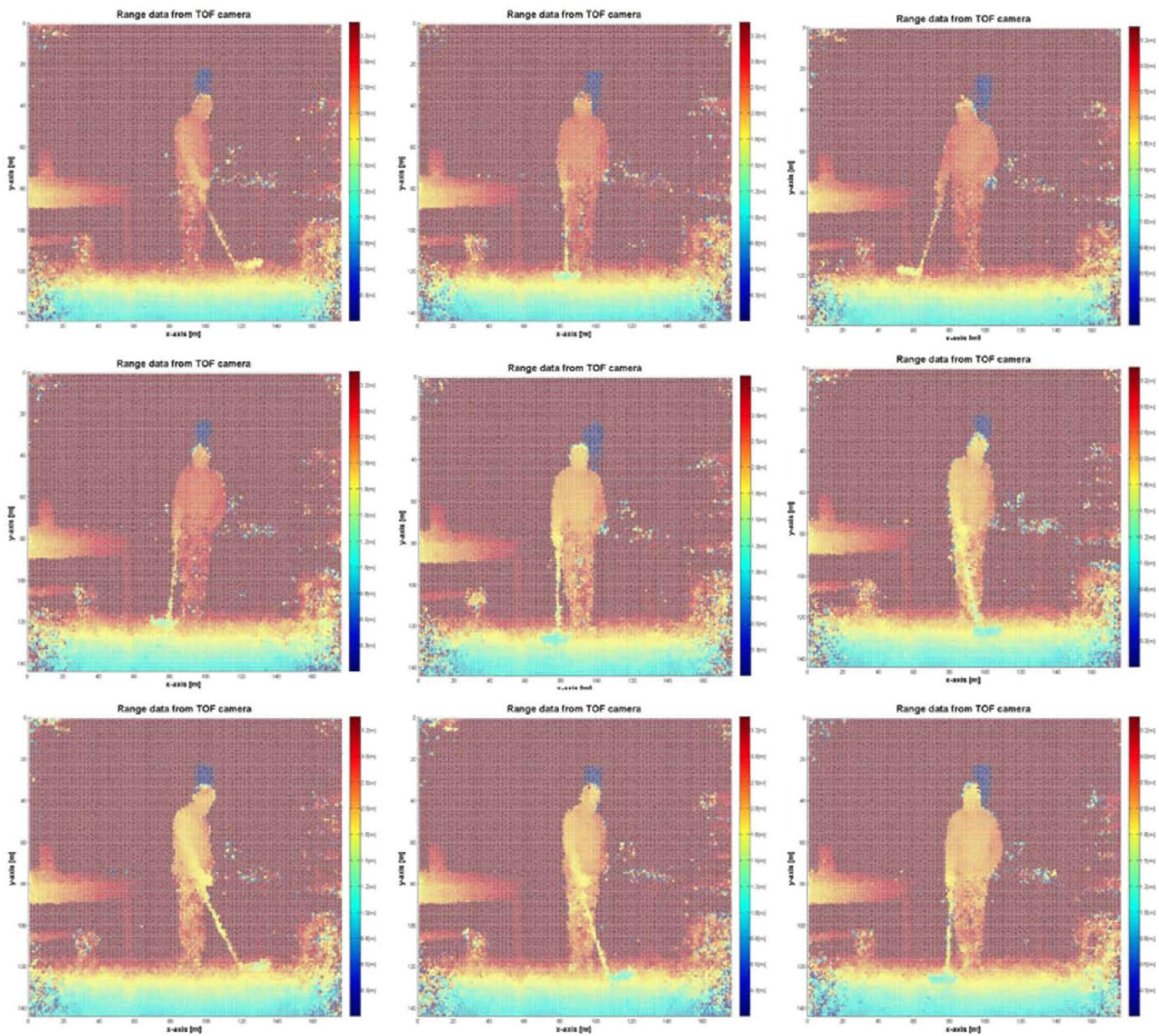

parallel to the surface. Roll angles of the search head have a very small variation, ranging from $-4^{\circ}$ to $4^{\circ}$.

The sweep coverage area, obtained from the data acquired with the motion trackers located in the hand-held detector, is shown in Figure 10. In this example, the total percentage of area swept is 97.9 percent. However, in this graph it is possible to observe that uneven sweep spacing is a major problem. To ensure the safety of the operator during the detection task, each sweep across the lane must overlap the previous one by about one-half the width of the detector head. This requirement is difficult to be achieved by novice operators that should keep in mind a spatial image of the last sweep motion carried out. The feedback provided by the training tool could be especially useful in this case, helping the novice operator to create the required mental patterns.
The sweep velocity of the search head during the scanning motions is shown in Figure 11. The goal was to hold a linear velocity lower than $0.3 \mathrm{~m} / \mathrm{s}$, that is considered the maximum limit velocity for detecting low-metal mines. Results show that the velocity is not constant during the sweeps. Maximum peak values take place in the middle of scan trajectories and the minimum at the ends, where the operator reduces the velocity before starting a new sweep in the opposite direction. It is also possible to note that after several sweeps, the operator starts to exceed the maximum recommended velocity limit. This can be a symptom of distraction or fatigue due to lack of training. Therefore, the usefulness of the tool for detecting these behaviours and implementing corrective actions afterwards also becomes highlighted.

Figure 12 shows the scan height variations above the ground of the search head determined from the data acquired 
Figure 9 Yaw, pitch and roll angles provided by the motion trackers
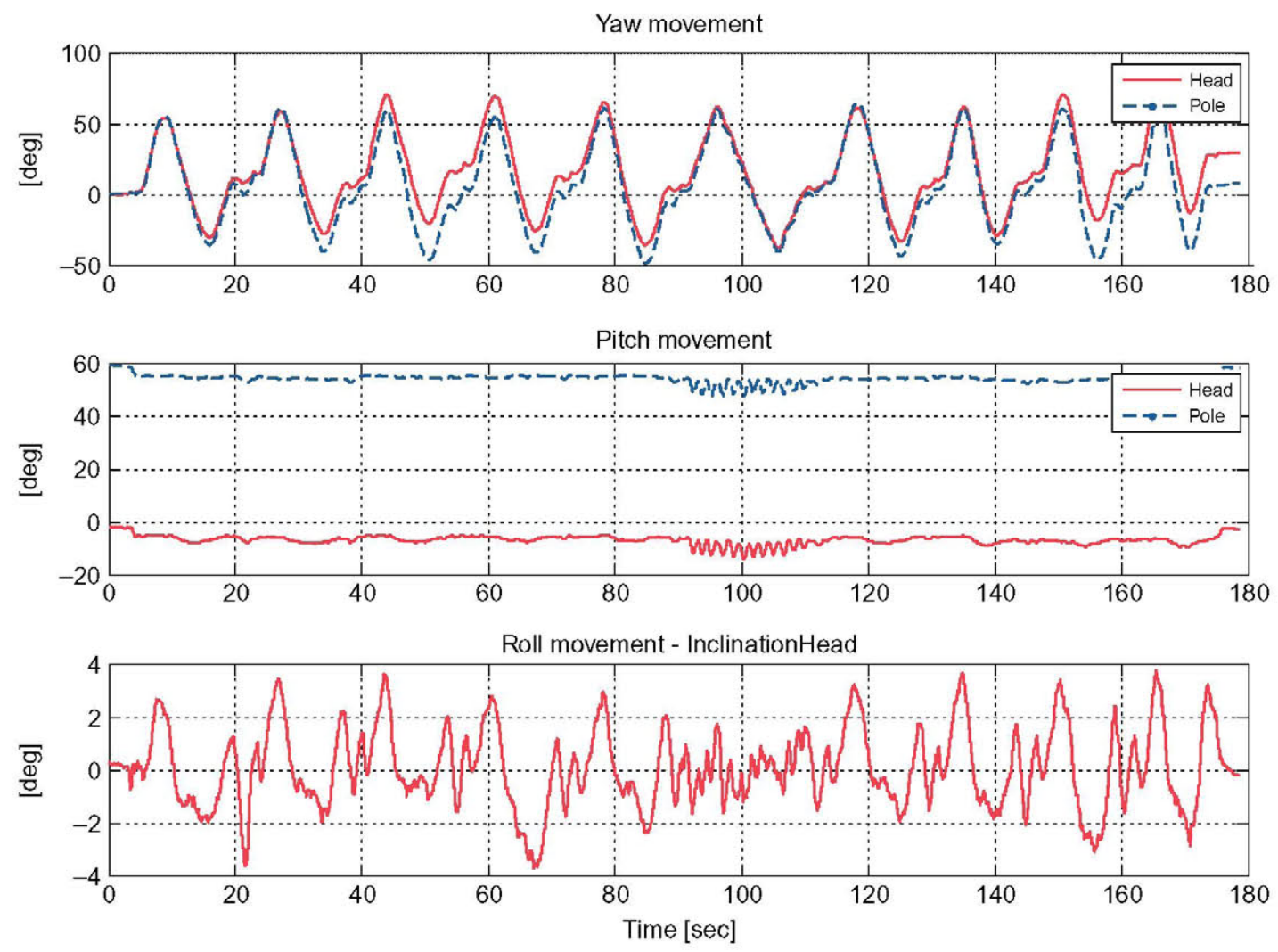

Figure 10 Sweep coverage area reconstruction using motion trackers

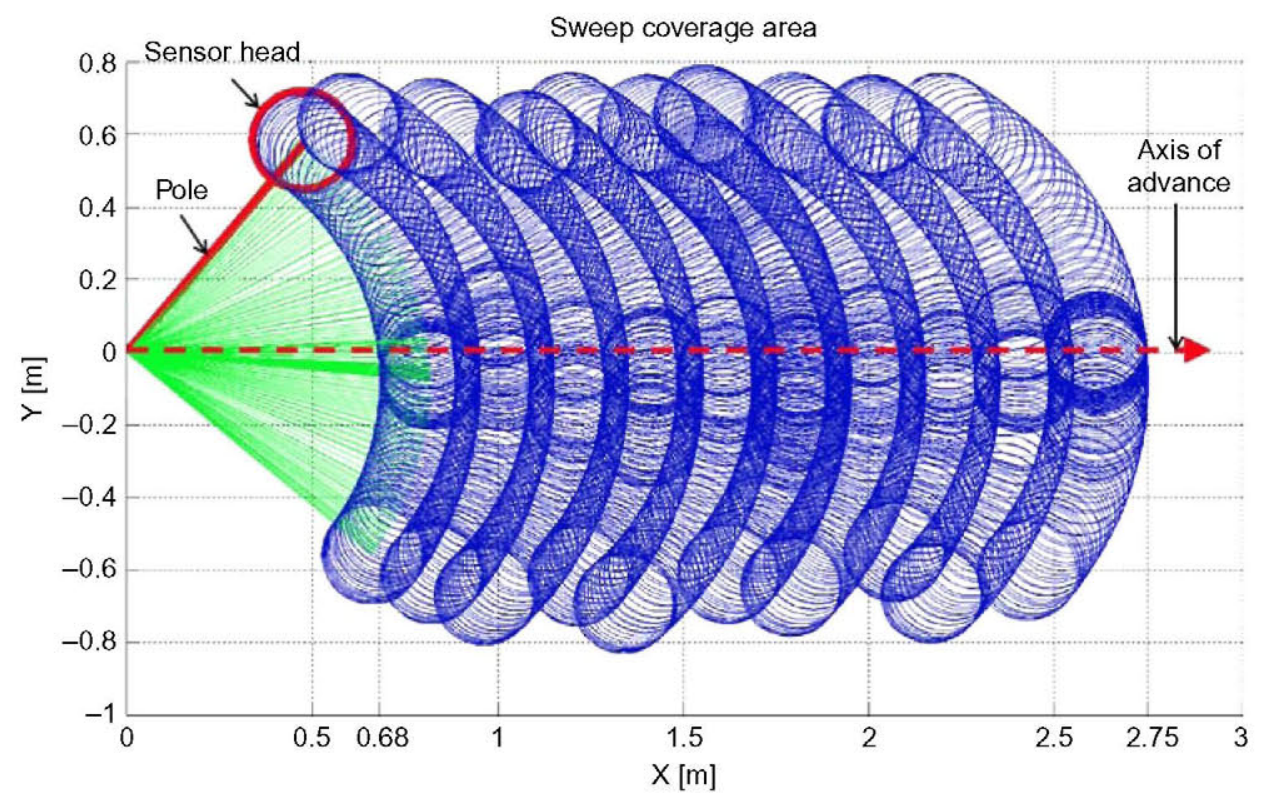

by the motion trackers. In this example, it is possible to observe that especially at the end of the sweeps, the operator has a tendency to raise the search head, reducing the detection depth. These height variations are expressed with respect to an initial reference position that was established on the flat horizontal ground. So if the training session is been carried out in a controlled environment where the surface profile is known, it is possible to use the motion trackers for obtaining the scan height. Otherwise, the omnivision system (Salinas et al., 2008) or the TOF camera should be utilised to monitor this variable. A network of rangefinders accommodated at the outer edge of the sensor-head frame 
Figure 11 Sweep velocity of the search head during the scanning motions

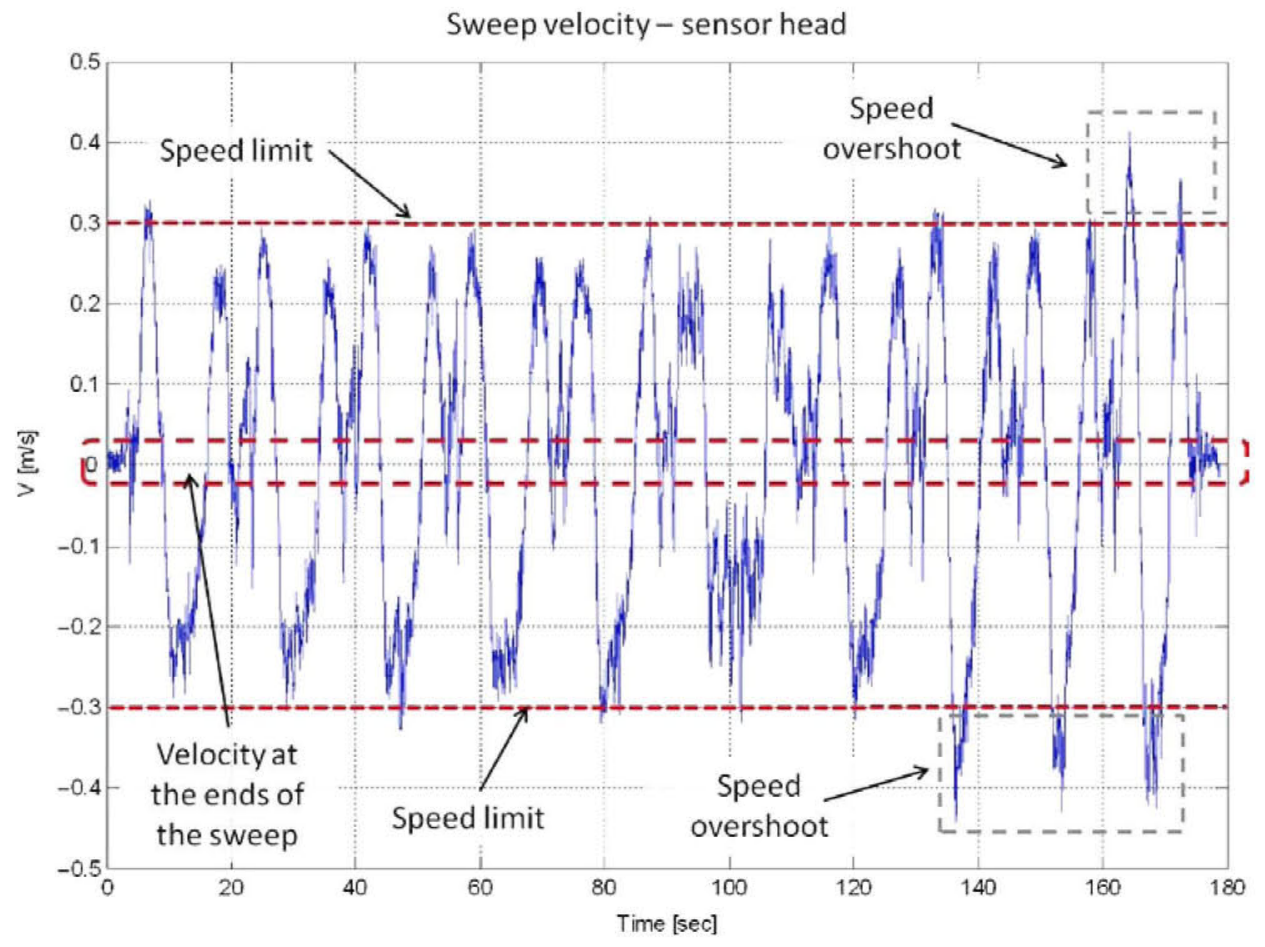

Figure 12 Scan height variations of the search head

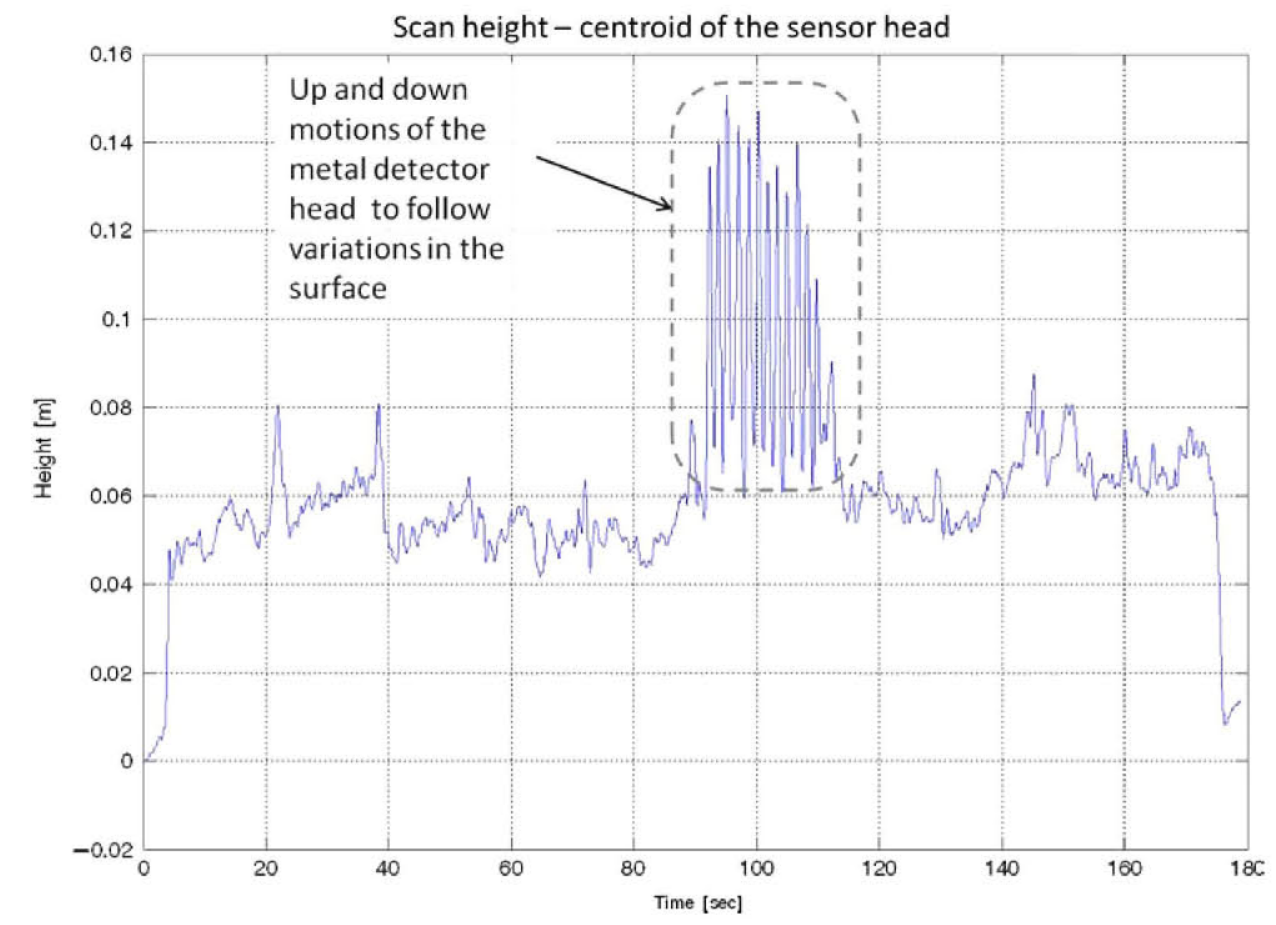

at regular intervals, pointing downwards at the ground could also provide data on the relative distance from the sensor head to the ground surface (Ponticelli et al., 2008; Ponticelli and Gonzalez de Santos, 2010). Finally, Figure 13 shows a real sequence carried out outdoors, highlighting the portability of the proposed system.

\section{Conclusions and future work}

In this work the design of a training tool for analysing with the final goal of improving the deminers' skills during close-in detection tasks with hand-held detectors was introduced and the testing of the elements that compose the sensory tracking system has been carried out. The sensory tracking approach 
Figure $13 \mathrm{~A}$ real sequence outdoors

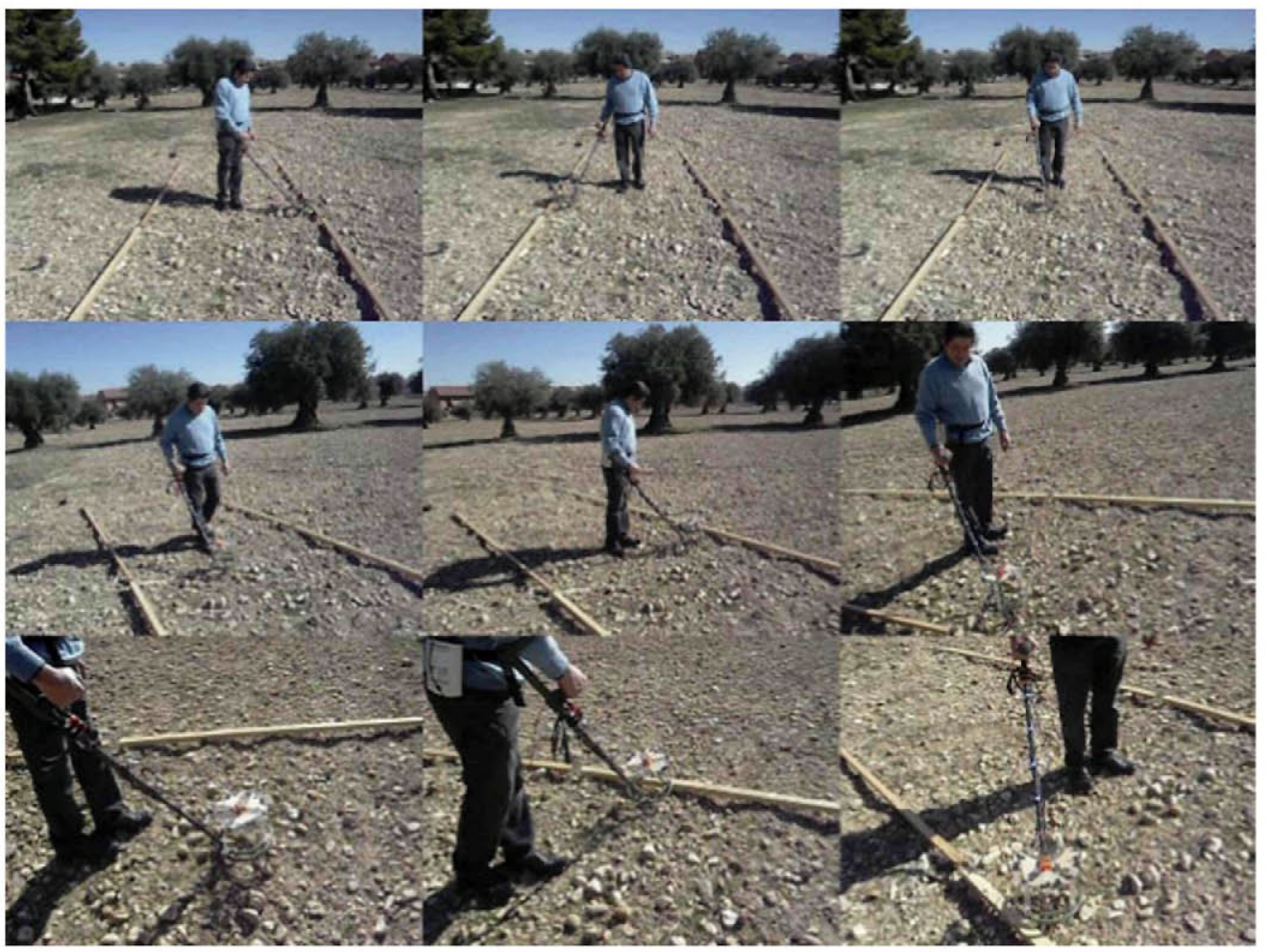

is based on an omnidirectional catadioptric stereo system, a TOF camera, two motion trackers and high-intensity LEDs utilized as landmarks for tracking the hand-held detector movements.

Both the catadioptric stereo system and the TOF camera are suitable for the detection and tracking of the hand-held detector motions in real time. The large FOV of the omnivision system enables tracking without the necessity of moving the cameras. It is also able to detect and track several hand-held detectors simultaneously. This feature can be very interesting to evaluate the performance of several operators and the interactions between them simultaneously, since interferences can occur while searching if the distance between the different search heads is less than $3 \mathrm{~m}$. The TOF camera provides depth maps of the scenes in a simpler way that the omnivision system, but can be affected by reflectivity object properties and aliasing effects when the camera-target distance overcomes the non-ambiguity range. Ergonomic or corporal behaviour analysis could be conducted with this equipment.

The motion trackers have been successfully utilised for determining the yaw and pitch movements of both the search head and the pole of the metal detector, the roll movement of the search head, the sweep coverage area and the swept velocity. Height variations of the search head expressed with respect to an initial reference can also be calculated. Its reduced size and weight and the existence of a wireless version of these devices make them much more appropriate to track the movements of the hand-held detector head in outdoor environments.

With the proposed sensory system, the experts' skills could be studied by quantifying some critical performance variables, so that they can be used later as reference values for the training task. Using the critical variables, the sensory tracking system, and a friendly human interface, the proposed system will be able to evaluate the efficiency of the deminers during the scanning tasks and to give them feedback on important information for improving their competencies.

There are still several issues that need to be addressed. One of them is the fusion of the omnivision system, the TOF camera and the inertial measurements in indoor environments. The analysis of the behavioural data from the experts using some standard exploratory techniques such as the PCA is also important in order to identify the relationship between some performance variables that may be hidden. Finally, an experimental training study has to be conducted in order to test the validity of the proposed tool as well as its utility for designing instructions in this field. The metrics for measuring the efficiency of the training could include the detection probability, the false alarm rate and the probability of false positives.

\section{References}

Baker, S. and Nayar, S.K. (1999), "A theory of single-viewpoint catadioptric image formation", International fournal of Computer Vision, Vol. 35 No. 2, pp. 175-96.

Baudoin, Y., Habib, M.K. and Doroftei, I. (2011), "Mobile robotics systems for humanitarian de-mining and risky interventions", in Baudoin, Y. and Habib, M.K. (Eds), Using Robots in Hazardous Environments, Woodhead Publishing Limited, Cambridge. 
Benosman, R. and Kang, S.B. (2001), Panoramic Vision: Sensors, Theory, and Applications, Springer, New York, NY.

Daniel, J., Hartman, P.E. and Dean, O.C. (2004), "New training tools: enhancing mine detection performance", The Role of the Military in Mine Action, Vol. 8 No. 1.

Daniels, D.J. and Curtis, P. (2006), "Minehound ${ }^{\mathrm{Th}}$ trials in Cambodia, Bosnia and Angola", Proceedings of the SPIE Defense and Security Conference 2006, Orlando, FL, Vol. 6217, $62172 \mathrm{~N}$

Daniels, D.J., Curtis, P., Hunt, N., Braunstein, J. and Merz, A. (2007), "Minehound ${ }^{\mathrm{TM}}$ transition to production", Proceedings of SPIE Conference on Detection and Remediation Technologies for Mines and Minelike Targets XII, Vol. 6553, $65531 \mathrm{~B}$.

Davison, A., Staszewski, J. and Boxley, G. (2001), "Improving soldier performance with the AN/PSS12", Engineer, Vol. 31, pp. 17-21.

Doheny, R.C., Burke, S., Cresci, R., Ngan, P. and Walls, R. (2005), "Hand-held standoff mine detection system (HSTAMIDS) field evaluation in Thailand", Proceedings of SPIE Conference on Detection and Remediation Technologies for Mines and Minelike Targets $X$, Orlando, FL.

Fernández, R., Salinas, C., Montes, H. and Armada, M. (2011), "Omnidirectional stereo tracking system for humanitarian demining training", Proceedings of the 8th International Symposium on "Humanitarian Demining 2011", Sibenik, Croatia.

GICHD (2006), Guidebook on Detection Technologies and Systems for Humanitarian Demining, Geneva International Centre for Humanitarian Demining, Geneva.

Gonzalez de Santos, P., Cobano, J.A., Garcia, E., Estremera, J. and Armada, M. (2007), "A six-legged robot-based system for humanitarian demining missions", Mechatronics, Vol. 17 No. 8, pp. 417-30.

Guelle, D., Smith, A., Lewis, A. and Bloodworth, T. (2003), Metal Detector Handbook for Humanitarian Demining, Office for Official Publications of the European Communities, Luxembourg.

Habib, M.K. (2002), "Mine clearance techniques and technologies for effective humanitarian demining", The fournal of ERW and Mine Action, Vol. 6 No. 1.

Habib, M.K. (2011), "Humanitarian demining mine detection and sensors", Proceedings of the 2011 IEEE International Symposium on Industrial Electronics, Gdansk, pp. 2237-42.

Hancock, R.A. (2006), "Decay in AN/PSS-14 operator skill at 30,60, 90 days following training", Landmine Detection Research Center Technical Report, Department of Social and Behavioral Sciences, Lincoln University, Jefferson City, MO.

Hegarty, M., Keehner, M., Cohen, C., Montello, D.R. and Lippa, Y. (2007), "The role of spatial cognition in medicine: applications for selecting and training professionals", in Allen, G. (Ed.), Applied Spatial Cognition, Lawrence Erlbaum Associates, Mahwah, NJ.

Herman, H. and Iglesias, D. (1999), "Human-in-theloop issues for demining", in Broach, T., Dubey, A.C., Dugan, R.E. and Harvey, J. (Eds), Proceedings of the SPIE Conference on Detection and
Remediation Technologies for Mines and Minelike Targets IV, Vol. 3710, pp. 797-805.

Herman, H., McMahill, J. and Kantor, G. (2000), “Training and performance assessment of landmine detector operator using motion tracking and virtual mine lane", in Dubey, A.C., Harvey, J.F., Broach, J.T. and Dugan, R.E. (Eds), Proceedings of the SPIE Conference on Detection and Remediation Technologies for Mines and Minelike Targets V, Vol. 4038, pp. 110-21.

Hussein, E.M. and Waller, E.J. (2000), "Landmine detection: the problem and the challenge", Applied Radiation and Isotopes, Vol. 53, pp. 557-63.

Landmine and Cluster Munition Monitor (2011), "Landmine monitor report", available at: www.the-monitor.org/index. $\mathrm{php} /$ publications/dsplay?url $=\mathrm{lm} / 2011 /$

Laura, D. (2011), "Training of personnel participating in humanitarian demining operations in the Republic of Croatia", Proceedings of the 8th International Symposium "Humanitarian Demining 2011", Šibenik, Croatia.

MacDonald, J. and Lockwood, J.R. (2003), Alternatives for Landmine Detection, Rand Publishing, Santa Monica, CA.

Miles, R.B., Dogariu, A. and Michael, J.B. (2012), "Bringing bombs to light", IEEE Spectrum, Vol. 49 No. 2, pp. 38-43.

Oggier, T., Lehmann, M., Kaufmann, R., Schweizer, M., Richter, M., Metzler, P., Lang, G., Lustenberger, F. and Blanc, N. (2004), "An all-solid-state optical range camera for $3 \mathrm{D}$ real-time imaging with sub-centimeter depth resolution (SwissRanger ${ }^{\text {TM }}$ )", in Mazuray, L., Rogers, P.J. and Wartmann, R. (Eds), Proceedings of SPIE Conference on Optical Design and Engineering, Vol. 5249, pp. 534-45.

Ponticelli, R. and Gonzalez de Santos, P. (2010), "Obtaining terrain maps and obstacle contours for terrain-recognition tasks", Mechatronics, Vol. 20 No. 2, pp. 236-50.

Ponticelli, R., Garcia, E., Gonzalez de Santos, P. and Armada, M. (2008), "A scanning robotic system for humanitarian de-mining activities", Industrial Robot, Vol. 35 No. 2, pp. 133-42.

Rosengard, U., Dolan, T., Miklush, D. and Samiei, M. (2001), "Humanitarian demining - nuclear techniques may help the search for landmines", IAEA Bulletin, pp. 16-19.

Salinas, C., Armada, M. and Gonzalez de Santos, P. (2008), "A new approach for terrain description in mobile robots for humanitarian demining missions", paper presented at the International Workshop on Robotics for Risky interventions and Surveillance of the Environment, Benicassim.

Salinas, C., Montes, H., Gonzalez de Santos, P. and Armada, M. (2011), "Catadioptric panoramic stereovision for humanoid robots", Robotica, pp. 1-13.

Sarter, N.B. and Amalberti, R. (2009), Cognitive Engineering in the Aviation Domain, Taylor \& Francis, Hillsdale, NJ.

Shimoi, N. (2002), Technology for Detecting and Clearing Landmines, Morikita Shuppan, Tokyo.

Staszewski, J.J. (1999), "Information processing analysis of human land mine detection skill", in Dubey, A.C., Harvey, J.F., Broach, J.T. and Dugan, R.E. (Eds), 
Proceedings of the SPIE Conference on Detection and Remediation Technologies for Mines and Minelike Targets IV, Vol. 3710, pp. 766-77.

Staszewski, J.J. (2004), "Models of expertise as blueprints cognitive engineering: applications to landmine detection", Proceedings of the 48th Annual Meeting of the Human Factors and Ergonomics Society, Vol. 48, pp. 458-62.

Staszewski, J.J. (2006), "Spatial thinking and the design of landmines detection training", in Allen, G.A. (Ed.), Applied Spatial Cognition: From Research to Cognitive Technology, Lawrence Erlbaum Associates, Mahwah, NJ, pp. 231-65.

Takahashi, K. and Gülle, D. (2010), "ITEP dual sensor test in Germany", paper presented at the International
Symposium on "Humanitarian Demining 2010", Šibenik, Croatia.

Zadeh, L. (1975), "The concept of a linguistic variable and its application to approximate reasoning - I", Information Sciences, Vol. 8 No. 3, pp. 199-249.

Zhihui, X., Wang, C. and Maojun, Z. (2008), "Catadioptric omni-directional stereo vision and its applications in moving objects detection", in Zhihui, X. (Ed.), Computer Vision, InTech, Vienna, p. 538.

\section{Corresponding author}

Roemi Fernández can be contacted at: roemi.fernandez@, car.upm-csic.es 